Cahiers de géographie du Québec

\title{
Les conceptions du Nord chez les géographes québécois
}

Une analyse comparée de quelques auteurs clés

Quebecer geographers' visions of the North

A compared analysis of a few key authors

\section{Las concepciones del Norte según los geógrafos quebequenses Un análisis comparativo de algunos autores claves}

\section{Martin SIMARD}

Volume 61, numéro 173, septembre 2017

La géographie québécoise : un regard particulier?

Version originale soumise en avril 2017. Version révisée reçue en janvier 2018.

URI : https://id.erudit.org/iderudit/1049372ar

DOI : https://doi.org/10.7202/1049372ar

Aller au sommaire du numéro

\section{Éditeur(s)}

Département de géographie de l’Université Laval

\section{ISSN}

0007-9766 (imprimé)

1708-8968 (numérique)

Découvrir la revue

\section{Citer cet article}

SIMARD, M. (2017). Les conceptions du Nord chez les géographes québécois : une analyse comparée de quelques auteurs clés. Cahiers de géographie du Québec, 61(173), 253-272. https://doi.org/10.7202/1049372ar
Résumé de l'article

Le Nord québécois est un milieu géographique complexe et relativement peu connu. L'adoption du Plan Nord (2011) a suscité un renouveau d'intérêt à son égard, malgré la pertinence relative et les aléas du projet. Néanmoins, des géographes québécois s'intéressent au Nord depuis plusieurs décennies. Ces travaux, dont le pionnier est incontestablement Louis-Edmond Hamelin, offrent différentes lectures du Nord québécois ou canadien. Celles-ci se déclinent selon la dimension épistémologique ou selon un angle plus sociopolitique. Nous voulons donc comparer les diverses conceptions du Nord de quatre auteurs reconnus : Louis-Edmond Hamelin, Christian Morissonneau, Jules Dufour et Caroline Desbiens. Ils s'inscrivent dans un continuum temporel sur plus d'un demi-siècle, bien que leurs écrits sur le Nord ne présentent pas de continuité parfaite sur le plan des idées. Le résultat de cette recherche offrira un panorama des écrits de géographes québécois sur un territoire géographiquement important, voire sur une caractéristique, la nordicité, qu'on retrouve à différents degrés sur l'ensemble du territoire québécois.
Tous droits réservés (C Cahiers de géographie du Québec, 2017
Ce document est protégé par la loi sur le droit d'auteur. L’utilisation des services d'Érudit (y compris la reproduction) est assujettie à sa politique d'utilisation que vous pouvez consulter en ligne.

https://apropos.erudit.org/fr/usagers/politique-dutilisation/ 


\title{
Les conceptions du Nord chez les géographes québécois.
}

\section{Une analyse comparée de quelques auteurs clés}

\author{
Quebecer geographers' visions of the North. \\ A compared analysis of a few key authors \\ Las concepciones del Norte según los \\ geógrafos quebequenses. Un análisis \\ comparativo de algunos autores claves
}

\author{
Martin SIMARD \\ Université du Québec à Chicoutimi \\ mgsimard@uqac.ca
}

\begin{abstract}
Résumé
Le Nord québécois est un milieu géographique complexe et relativement peu connu. L’adoption du Plan Nord (2011) a suscité un renouveau d'intérêt à son égard, malgré la pertinence relative et les aléas du projet. Néanmoins, des géographes québécois s'intéressent au Nord depuis plusieurs décennies. Ces travaux, dont le pionnier est incontestablement Louis-Edmond Hamelin, offrent différentes lectures du Nord québécois ou canadien. Celles-ci se déclinent selon la dimension épistémologique ou selon un angle plus sociopolitique. Nous voulons donc comparer les diverses conceptions du Nord de quatre auteurs reconnus : Louis-Edmond Hamelin, Christian Morissonneau, Jules Dufour et Caroline Desbiens. Ils s'inscrivent dans un continuum temporel sur plus d'un demi-siècle, bien que leurs écrits sur le Nord ne présentent pas de continuité parfaite sur le plan des idées. Le résultat de cette recherche offrira un panorama des écrits de géographes québécois sur un territoire géographiquement important, voire sur une caractéristique, la nordicité, qu'on retrouve à différents degrés sur l'ensemble du territoire québécois.
\end{abstract}

\section{Mots-clés}

Conceptions, territoire, Nord, géographes, Québec.

\begin{abstract}
Northern Quebec is a complex geographical environment which is still relatively unknown. Quebec's Plan Nord (2011) has brought a renewed interest in the region, despite the ups and downs of the project. Nevertheless, Quebec geographers have been interested in the North for several decades. Their work, pioneered by Louis-Edmond Hamelin, offers different readings of Northern Quebec and Canada. Their writings can be analyzed through an epistemological dimension or a sociopolitical angle. The aim of this research is to compare the different visions of four well-known authors: Louis-Edmond Hamelin, Christian Morissonneau, Jules Dufour, and Caroline Desbiens. They all seem to be part of a time continuum that spreads over half a century, although their writings on the North present a range of approaches and ideas. The results will offer a panorama of their writings on a massive piece of land in Quebec, and on a particularity: nordicity, which can be found at different levels across the province.
\end{abstract}

\section{Keywords}

Vision, territory, North, geographers, Quebec. 


\section{Resumen}

El Norte quebequense constituye un medio geográfico complejo, poco conocido. La adopción del Plan Nord (2011) ha creado un nuevo interés, pese a su relativa pertinencia y a sus vicisitudes. Sin embargo, desde hace varias décadas, los geógrafos quebequenses se interesan del Norte. Entre ellos, Luis-Edmond Hamelin es incontestablemente el pionero. Sus trabajos ofrecen diferentes lecturas del Norte quebequense o canadiense, ya sea según la dimensión epistemológica o según un enfoque sociopolítico. En la presente investigación comparamos diversas concepciones del Norte según cuatro autores destacados: Luis-Edmond Hamelin, Christian Morissonneau, Jules Dufour y Caroline Desbiens que se sitúan a lo largo de un continuum de más de medio siglo, aunque esos escritos sobre el Norte no presenten continuidad perfecta en el plano de las ideas. Como resultado de la investigación ofrecemos una variedad de textos escritos por geógrafos quebequenses a cerca de un territorio geográficamente importante, sea sobre una característica, la nordicidad, presente en grados diferentes sobre todo el territorio quebequense.

\section{Palabras claves}

Concepciones, territorio, Norte, geógrafos, Quebec.

\section{Introduction}

Le Nord québécois est un milieu géographique vaste, complexe et relativement peu connu, encore aujourd'hui. L’adoption successive par le gouvernement du Québec des Plans Nord I et II, en 2011 et 2015, a suscité un renouveau d'intérêt à l'égard de ce territoire singulier, malgré la pertinence relative du projet et ses aléas. Les géographes québécois s'intéressent au Nord depuis plusieurs décennies, à l'instar de nombreux anthropologues, sociologues, historiens ou géologues (Harvey, 1994). Ces travaux de géographes illustrent différentes visions du Nord québécois, ou du Nord canadien dans son ensemble. Par leur ampleur et une certaine continuité sur le plan des idées, ils constituent un élément important de la géographie humaine québécoise. Ces conceptions géographiques du Nord s'inspirent des voyages et des écrits d'explorateurs qui agissent comme témoins de paysages et de réalités socioculturelles inconnues des Euro-Canadiens (Duhaime, 2010) pour évoluer vers des thématiques particulières comme l'exploitation des ressources, les processus d'évolution des milieux naturels, de même que la santé et les aspirations des Autochtones (Allard et Lemay, 2013). Les recherches se déclinent également selon leur dimension épistémologique (approches positiviste, humaniste ou critique) ou selon leur ascendant sociopolitique (visions nationaliste, économiciste, environnementaliste ou axée sur les conditions de vie et les droits des Autochtones). ${ }^{1}$

Avant d'aborder ces diverses lectures géographiques, il convient de tenter de définir ce Nord québécois qui évolue non seulement dans les esprits mais sur le plan légal, avec les extensions de 1898 et 1912 et le retranchement de 1927. À travers le temps

1 Le terme nationalisme, fortement péjoratif en Europe, est largement utilisé au Québec pour décrire les désirs d'émancipation socioéconomique et politique des Québécois, désirs généralement soutenus par le gouvernement du Québec. Le terme n'est pas nécessairement synonyme d'appui à l'indépendance du Québec et il se décline en différents degrés (nationalistes durs, modérés, voire mous). Le nationalisme québécois se veut habituellement inclusif et territorial même s'il interpelle davantage les Québécois francophones, lesquels ont été considérés comme des citoyens de seconde classe, de la Conquête britannique jusque dans les années 1960. 
et les auteurs, le Nord peut être conçu comme étant le massif laurentien au nord de Montréal, les basses-terres de l'Abitibi et du Lac-Saint-Jean, la côte du Labrador, la Baie-James ou le pays inuit. Quelle que soit sa localisation réelle ou imaginée, le Nord, la nature sauvage et l'Arctique deviennent de véritables symboles nationaux aux yeux de bien des Québécois et Canadiens, du XIXe siècle et du début du XXe (Bailly et al., 1992). Cette symbolique du Nord, version nord-américaine, semble rejoindre un mythe nordique présent en Europe, dans la curiosité à l'égard de la Scandinavie jusqu'à l'obsession pour le passage du Nord-Ouest (Droit, 2014). Les conceptions fantasmées ou mythiques du Nord semblent toujours vivantes au sein de la population et chez les gouvernants, au Québec. Les géographes s'intéressent de plus en plus à ces symboles et à ces mythes.

Cela étant posé, cet article recensera, décrira et comparera ces diverses conceptions du Nord qui dominent chez les géographes québécois qu'on peut qualifier de modernes, c'est-à-dire ceux et celles de la deuxième moitié du XXe siècle jusqu'au début du XXIe. Cet exercice se fera à l'aide d'une brève analyse des principaux textes de quatre auteurs reconnus, abordés ici dans un ordre chronologique ou générationnel: LouisEdmond Hamelin, Christian Morissonneau, Jules Dufour et Caroline Desbiens. Ces quatre géographes du Québec s'inscrivent dans un continuum temporel, bien que leurs écrits sur le Nord ne présentent pas nécessairement de continuité parfaite sur le plan des idées. Le résultat de cette recherche offrira un panorama des écrits de géographes québécois sur un territoire géographiquement important, voire sur l'une de ses caractéristiques principales, la nordicité, qui s’applique à différents degrés à l'entièreté du territoire québécois.

Nous débuterons par une description de la méthode mise en œuvre dans notre recherche. Étant donné que certains auteurs ciblés ont énormément publié, en particulier Louis-Edmond Hamelin, nous nous concentrerons sur deux ou trois œuvres maîtresses pour chacun des chercheurs, tout en tenant compte des virages importants qui pourraient avoir eu lieu entre leurs premiers écrits et les publications récentes. Deuxièmement, nous présenterons les quatre auteurs et discuterons leurs principales idées à tour de rôle. Cette démarche, qui peut sembler rigide, s'impose, à notre avis. En effet, les propos des auteurs doivent être mis en relation avec le profil de l'auteur et l'époque de ses écrits. De plus, les idées et affirmations des universitaires sélectionnés se juxtaposent et se télescopent, pouvant difficilement être extraites de leur contexte pour être traitées de manière thématique. En troisième lieu, nous tenterons de mettre en discussion et de comparer les visions de ces auteurs nordistes québécois.

\section{Méthodologie}

En premier lieu, mentionnons que les quatre géographes sélectionnés l'ont été sur la base des critères suivants: leur reconnaissance dans le milieu des géographes, le rayonnement de leur œuvre, de manière générale, ainsi que l'ampleur et la durée de leurs travaux et études sur le Nord. Il faut dire que les géographes s'intéressant significativement au Québec du Nord sont relativement peu nombreux et qu'il s'agit presque exclusivement d'hommes. ${ }^{2}$ Certes, nous aurions pu considérer les travaux

2 La faible présence des femmes en géographie humaine du Nord, au Québec, rend malheureusement impossible un échantillonnage ou une analyse des idées sur la base des sexes. 
de Jean Morisset (1985), mais ils demeurent marginaux et radicaux sur de multiples éléments de contenu (Pelletier, 1986). Ceux d'Henri Dorion (Dorion et Lacasse, 2011) ou de Frédéric Lasserre (2003) auraient également pu être considérés, mais ils portent surtout sur les questions de frontières et de géopolitique, révélant principalement le Nord québécois comme un espace administratif contesté. ${ }^{3}$ Finalement, les recherches de Michel Allard et d'autres chercheurs du Centre d'études nordiques (CEN) de l'Université Laval auraient aussi pu constitué matière à réflexion, mais ce sont des travaux de terrain (2013). Des plus pertinents socialement et scientifiquement, ces travaux sont généralement techniques ou très appliqués, parlant essentiellement de pergélisol, de précipitations ou de changements climatiques, quoiqu'ils ne soient pas neutres. En effet, ces travaux contribuent indirectement à forger une conception utilitaire du Nord comme bassin de ressources à explorer, à maîtriser, à exploiter.

En ce qui concerne les œuvres étudiées, il s'agira de Nordicité canadienne (1975), Le Nord et l'hiver dans l'hémisphère boréal (2000) et L'âme de la terre. Parcours d'un géographe (2006), pour Louis-Edmond Hamelin. Dans le cas de Christian Morissonneau, nous nous pencherons sur La terre promise: le mythe du Nord québécois (1978) et un court texte de 1982 s'intitulant Le Nord qui est la nature qui est la féminitude. Eu égard à Jules Dufour, la situation est plus difficile, car il ne compte pas de livre portant spécifiquement sur le Nord, mis à part un atlas qu'il a codirigé pour un organisme gouvernemental et dont le style est plutôt terne (Dufour et Côté, 1983). Toutefois, Dufour traite du Nord à travers la question des Autochtones, notamment dans deux textes parus dans les Cahiers de géographie du Québec, en 1993 et 1996. Ces textes nous serviront de matériaux. Pour finir, la contribution aux études nordiques de Caroline Desbiens sera analysée à partir du livre Puissance Nord. Territoire, identité et culture de l'hydro-électricité au Québec (2015). Nous examinerons également l'un de ses textes, également paru dans les Cahiers de géographie: 10 idées pour le Nord: un manifeste pour la nordicité (2012).

Finalement, rappelons que nous cherchons à saisir les conceptions du Nord de ces quatre géographes et non leur interprétation de la vision d'autres auteurs ou acteurs sociaux. En effet, certains d'entre eux, nommément Morissonneau et Desbiens, ont analysé les visions d'autres individus ou catégories de personnes (curés colonisateurs, politiciens, Autochtones, etc.), ce qui peut rendre plus complexe le repérage de leur propre conception. Néanmoins, nous tenterons de distinguer leur vision de géographe à travers leurs réactions ou commentaires personnels face aux conceptions du Nord de ceux et celles qu'ils auscultent. Par ailleurs, il eût été intéressant d'étudier les approches ou travaux sur le Nord d'autres auteurs non géographes, notamment les sociologues, les anthropologues ou les littéraires, par exemple les diverses recherches effectuées par Thibault Martin $(2003$; 2010) ou Gérard Duhaime (Duhaime et Godmaire, 2002 ; Duhaime et Robichaud, 2010) ou le chantier récent lancé par Daniel Chartier de la Chaire de recherche sur l'imaginaire du Nord, de l'hiver et de l'Arctique de l'UQAM. Des géographes francophones non québécois auraient également pu être cités, en particulier Éric Canobbio (2009). Il reste que le présent travail s’inscrit dans un projet d'écriture ciblant précisément les géographes et la géographie du Québec; les contributions de ces intellectuels de l'extérieur du Québec ne seront donc pas abordées, malgré leur grande valeur.

3 Notez que Frédéric Lasserre a également écrit sur le dossier de la Baie-James (Lasserre, 2009). 


\section{Le Nord et la nordicité chez Louis-Edmond Hamelin (1923-)}

Louis-Edmond Hamelin peut être considéré comme l'un des pionniers, voire comme l'un des «pères» de la géographie québécoise, à l'instar de Benoît Brouillette et de Pierre Dagenais, dans le contexte d'une géographie institutionnalisée qui prenait forme au milieu du XXe siècle. Ayant été l'élève de Raoul Blanchard à Grenoble, Hamelin jouera le rôle de passeur entre la géographie française et la géographie québécoise. Cela ne l'empêchera pas de parcourir et d'étudier l'ensemble de la zone circumpolaire et d'intervenir sur la scène géographique canadienne, tout en étant rattaché à l'Université Laval, de Québec, et au CEN, qu’il a fondé en 1961. Dans cette foulée, il a été président de l’Association canadienne des géographes en 1971 et 1972.

C’est en 1975 que Louis-Edmond Hamelin publie Nordicité canadienne. Ce livre, qui sera traduit en anglais en 1979, recevra le prix du Gouverneur général du Canada, en 1976. Il s'agit maintenant d'un grand classique de la géographie québécoise et canadienne qui a contribué à consolider le Nord ou la nordicité comme des référents importants de l'identité nationale, en particulier, et paradoxalement, au Canada anglais. Notre analyse portera principalement sur ce texte. Dès le départ, il est important de remettre le livre dans son cadre historique, soit celui du début des années 1970. Les Québécois et les Canadiens connaissent alors peu le Nord. Le projet de la Baie-James débute à peine et la Convention de la Baie-James et du Nord québécois (CBJNQ) n’est pas en vigueur (1975). Le gouvernement québécois est toujours faiblement présent dans le Nord de la province, notamment au Nunavik, territoire qu'on désigne à l'époque comme étant le Nouveau-Québec. À l'exception de l'aval donné à l'exploitation du fer dans la région de Schefferville, au début des années 1950, il y a peu de signes d'appropriation du Nord par l'État québécois. Finalement, à l'époque où Hamelin écrit l'essentiel de son œuvre, le Nunavut n'existe pas (1999), la Paix des braves n'est pas encore signée avec les Cris (2002), le Plan Nord n'est pas formalisé (2011) et les rares interventions de l'État québécois relèvent d’une logique éclatée et teintée de paternalisme, du moins aux yeux d'Hamelin (1975).

Nordicité canadienne stimule l'intérêt du lecteur en présentant d'abord le Nord comme vaste et diversifié. Le livre souligne les difficultés socioéconomiques des Autochtones, leur dépendance face aux «Blancs» et au gouvernement fédéral, situation qu’Hamelin qualifie de coloniale. Il regrette que la majorité des gens du Sud connaissent peu ou pas le Nord et qu'ils y associent des propriétés plutôt négatives. Par opposition, il décrit la vision sensible du territoire que possèdent les Autochtones, une vision marquée par leurs relations au milieu naturel, le cycle des saisons et leur volonté de s'adapter au milieu. Il mentionne le rôle significatif de la toponymie pour eux et le caractère inapproprié des noms de lieux imposés par les Canadiens du Sud. Il décrit la territorialité autochtone comme différente de celle des Occidentaux, mais il voit déjà poindre, par nécessité ou par contagion des pratiques des allochtones, l'enjeu des revendications territoriales et le besoin de frontières si largement répandu à travers le monde.

Deux éléments majeurs ressortent de ce texte: Hamelin y propose le terme nordicité et il suggère des délimitations externes et internes du Nord en fonction de différents critères. Au sujet du terme nordicité, il le définit brièvement au début de l'ouvrage comme «l'état et le niveau polaire dans l'hémisphère boréal» (Hamelin, 1975: 11), un phénomène qu'il décrit comme variable, mesurable et évoluant dans le temps. Cette définition, plutôt limitée, sera complétée plus loin dans l'ouvrage à l'aide du sous- 
concept de nordicité mentale, c'est-à-dire la nordicité comme état d'esprit caractérisé par une vision positive du froid et de la neige, une ouverture aux autres cultures, une conception différente de la distance et du temps, une mise de côté des préjugés à caractère racial, un abandon des visées militaristes et géopolitiques, etc. Dans son texte de 2000 analysé ici, Hamelin souligne tout spécialement la souplesse et le caractère englobant du terme nordicité. Il rappelle également les multiples déclinaisons possibles apparues avec le temps : nordicité zonale, saisonnière, développementale, normative, totale, etc.

Le troisième élément d'importance associé à l'ambitieux projet de Louis-Edmond Hamelin est de mieux faire connaître le Nord à travers la délimitation et la zonation des milieux nordiques (voir figure 1). Cette démarche, très cartésienne et qui semble s'opposer aux propos humanistes ultérieurs du géographe, serait nécessaire, selon lui, afin de mieux saisir la diversité des situations géographiques et discerner les zones tempérées affectées par des hivers plus ou moins longs, du Nord véritable, domaine du froid persistant, de la distance, de la basse densité de population, d'une autochtonie majoritaire et d'une plus grande authenticité des cultures amérindiennes et inuit. Sur cette base, Hamelin détermine 10 critères pouvant être quantifiés afin d'attribuer une "valeur polaire» (VAPO) à chaque lieu et, éventuellement, permettre une zonation des territoires nordiques (tableau 1). Le $45^{\mathrm{e}}$ parallèle nord est alors fixé comme limite sud à ne point franchir, indépendamment des effets orographiques au sein des zones plus méridionales de l'hémisphère Nord, et le pôle Nord est défini comme la limite absolue, en toute logique.

Laissons Hamelin décrire lui-même sa démarche:

Chaque facteur indique, pour un lieu ou une circonstance, une certaine quantité de valeurs polaires (VAPOS) dont, par définition, le nombre est inférieur à 100. La somme des indications nordicistes portées par chaque facteur fournit la masse Nordique ponctuelle. Si, en un poste, chacun des dix critères correspond à 100 points, le total s'établirait à 1000 , soit une Nordicité d'intensité maximale, en fait celle du pôle Nord. Un VAPO correspond au millième de la nordicité géographique au pôle. Un pas de plus consiste à réunir sur une carte les lieux qui possèdent le même nombre d'unités, c'est-à-dire la même nordicité; du coup, apparaissent des traits appelés isonords (2000: 9).

\section{Tableau 1 Critères d'Hamelin pour établir la nordicité d'un lieu}

\begin{tabular}{|c|c|c|}
\hline Critère & Type & Repères pour la quantification \\
\hline Latitude & Localisation & $0=45^{\circ}$ nord $/ 100=90^{\circ}$ nord \\
\hline Chaleur estivale & Biophysique & $0=150$ jours à $+5,6^{\circ} \mathrm{C} / 100=0$ jour à $+5,6^{\circ} \mathrm{C}$. \\
\hline Froid annuel & Biophysique & $0=-$ de $1000 \mathrm{j} . /{ }^{\mathrm{o}}+$ de $0^{\circ} \mathrm{C} / 100=+$ de $12000 \mathrm{j} . /{ }^{\circ}+$ de $0^{\circ} \mathrm{C}$. \\
\hline Type de glace & Biophysique & 0=gélisol pendant - de 1 mois / 100=pergélisol continu \\
\hline Précipitations & Biophysique & $0=$ pluie de + de $500 \mathrm{~mm} / 100=$ pluie de - de $100 \mathrm{~mm}$ \\
\hline Végétation & Biophysique & $0=$ forêt dense $/ 100=$ désert pierreux \\
\hline Accès non aérien & Socioéconomique & $0=$ accès par bateau ou route $/ 100=$ aucun accès \\
\hline Accès aérien & Socioéconomique & $0=$ service quotidien $/ 100=$ aucun service à proximité \\
\hline Population & Socioéconomique & $0=$ plus de $5000 \mathrm{~h}$. ou $4 \mathrm{~h} . / \mathrm{km}^{2} / 100=$ aucun h. ou 0 h. $/ \mathrm{km}^{2}$ \\
\hline Économie & Socioéconomique & $0=$ services multiples $/ 100=$ aucune production \\
\hline
\end{tabular}

Sources: Hamelin, 1975: 84-88; 2000:10 
Figure 1 Zonation du Nord selon Hamelin (isonords de 200, 300 et 500 VAPOS)

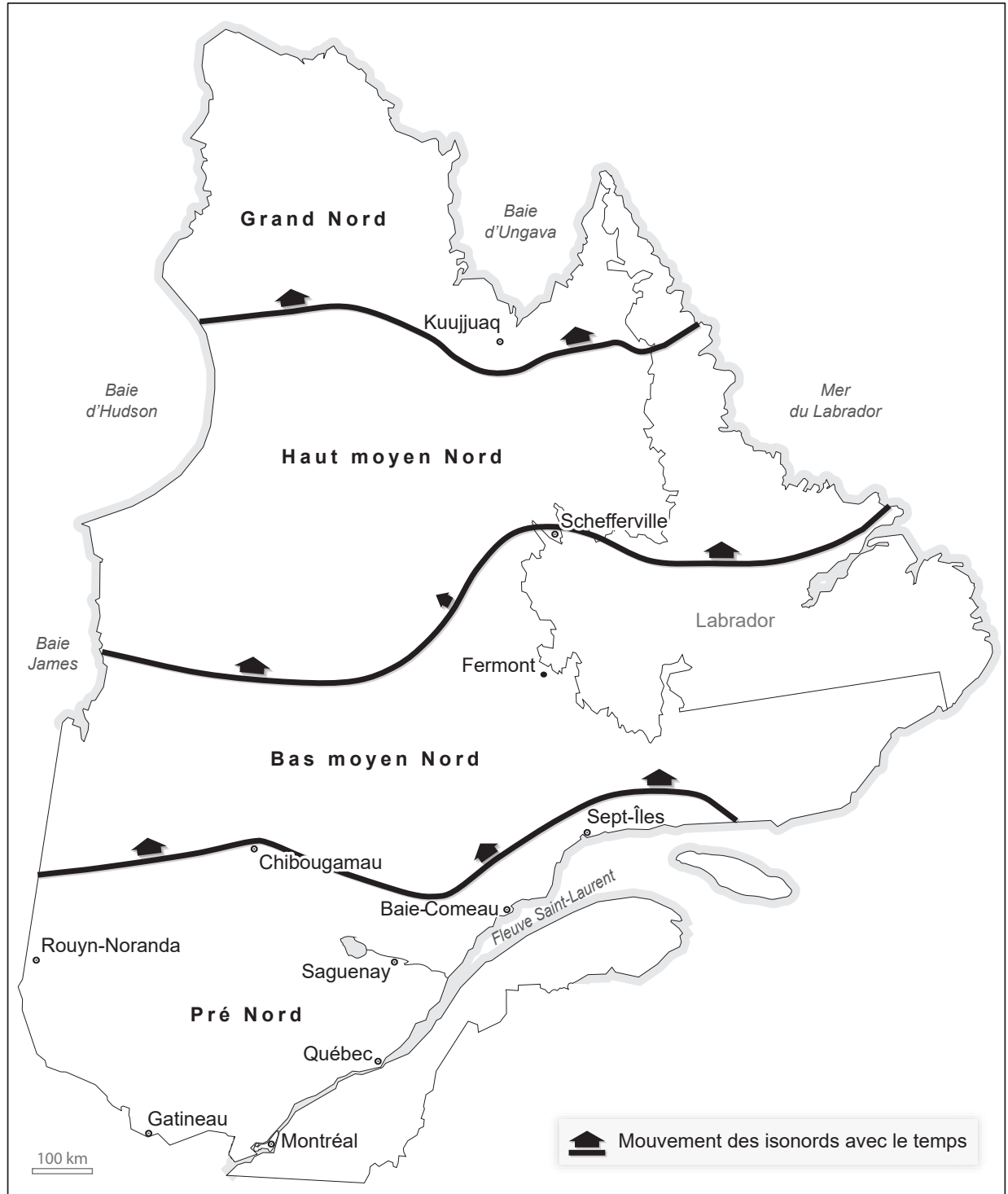

Conception : Brisson, 2016 (LERGA, UQAC)

Source: Hamelin, 1973

Les travaux de Louis-Edmond Hamelin restent influents dans le champ des études nordiques malgré le vieillissement progressif de certains éléments quantitatifs. Ses nombreux ouvrages ont notamment contribué à faire connaître le Nord, à le catégoriser par sous-régions de même qu’à enrichir le vocabulaire géographique, par exemple, avec la création du terme glaciel pour décrire les glaces flottantes. Cependant, on peut regretter qu'aucun chercheur des générations suivantes n’ait cru bon mettre à jour le calcul des VAPOS et la cartographie qui en résulte. Il est vrai qu'il s'agirait d'une tâche colossale, mais l'essentiel des travaux sur ce sujet date des années 1960, ce qui 
justifierait, à notre avis, un renouvellement des données sinon des variables et des indicateurs de sa méthode. Il est aussi permis de s’interroger sur les seuils sélectionnés pour chacun des critères retenus, notamment en ce qui concerne la population totale des établissements nordiques. Dans la même optique, les changements climatiques, l'évolution démographique et le développement des infrastructures de transport et de communication militent en faveur d'une mise à jour, cela, dans la mesure où cette régionalisation du Nord serait jugée nécessaire.

Sans rejeter les travaux d'Hamelin sur la délimitation du Nord, Fernand Harvey rappelle l'interprétation plus traditionnelle et plus simple défendue par l'historien Morton (1970), soit la frontière de la colonisation agricole. Une autre critique, soulevée par le géographe Robert M. Bone (2016), a trait au postulat de base de la classification d'Hamelin: plus le Nord se développe (routes, populations, etc.), plus il perd sa nordicité. On peut interpréter cela comme une volonté altruiste ou «pro-autochtone» d'Hamelin à ce que le Nord soit enfin considéré comme un territoire autochtone authentique ou, à l'opposé, qu'il demeure lié à une association très «sudiste» entre sous-développement et nordicité. Hamelin semble répondre lui-même à cette critique en soutenant plus tard que «la nordicité implique de penser et de construire le Nord autrement que les non-Autochtones l'ont fait» (2006: 75). Cependant, il faut bien admettre qu'il serait difficile de limiter les routes, l'accès aérien et les activités économiques dans cet éventuel développement alternatif du Nord. Donc, faut-il comprendre que les Autochtones sont condamnés à se «dénordifier» eux-mêmes par leur insertion partielle ou complète dans la modernité?

In fine, au-delà de l'attention portée à sa cartographie du Nord, il faut aussi comprendre que les recherches d'Hamelin s'articulaient en fonction d'une approche scientifique positiviste fondée sur l'analyse spatiale et la modélisation, pour ensuite évoluer rapidement vers une approche humaniste, en particulier avec l'élaboration d'un nouveau vocabulaire pour décrire le Nord et ses attributs. Il y a ainsi tout un monde entre Nordicité canadienne (1975) et l'Âme de la terre (2006). Cette seconde approche plus humaniste a, à notre avis, donné le ton aux recherches subséquentes en géographie humaine sur le Nord du Québec, comme en témoignent les trois géographes suivants, à l'intérieur de notre échantillon. Sur le plan idéologique ou politique, les propos d'Hamelin ne semblent pas motivés par le nationalisme québécois ou canadien, mais ils aspirent visiblement à une meilleure intégration socioéconomique et politique des espaces nordiques aux cadres étatiques existants, dans le respect des particularités de ces milieux. De la sorte, Hamelin utilise occasionnellement l'expression «Québec total».

\section{Le mythe du Nord selon Christian Morissonneau (1943-)}

La terre promise: le mythe du Nord québécois est paru en 1978, c'est-à-dire quelques années après le Nordicité canadienne d'Hamelin, auquel il ne fera par ailleurs aucune référence, mais dont il traitera brièvement dans un texte subséquent, en 1982. Morissonneau, qui se définit également comme historien, est assurément le géographe le plus difficile à décoder. En effet, il dévoile peu sa vision du Nord, tout occupé qu'il est à révéler le mythe du Nord construit par les élites du début du XXe siècle. Malgré tout, à travers les lignes, il nous semble percevoir le Nord comme un espace 
périphérique, largement inhabité et peu propice à l'agriculture. Cela dit, Il s'intéresse surtout au Pré-Nord ou au Moyen Nord et il dénonce très tôt le rabaissement des Autochtones au titre de data dans des études d'impact (1982: 245).

Comme le titre de son ouvrage l'indique, et à la différence d'Hamelin, Christian Morissonneau se penche sur le Québec plutôt que sur l'ensemble canadien. En outre, son approche relève principalement de l'histoire et de la sociologie, malgré son objet à caractère géographique. De plus, son analyse plutôt théorique et nationaliste se focalise sur le thème du mythe, qu'il décrit comme le noyau dur des idéologies colonisatrices et comme un élément de la culture, au Québec. Pour lui, ce mythe explique «à la fois comment et pourquoi les choses se sont passées et comment elles doivent se passer » (1978: 8). Contrairement à Hamelin, dont la conception territoriale se reflète dans les caractéristiques concrètes du Nord associées à ses VAPOS, et à partir d'une vision sensible inspirée des cultures autochtones, Morissonneau présente essentiellement le Nord comme une construction sociale façonnée par les élites canadiennes-françaises, dans une optique de survivance face aux défis du XIXe siècle (Acte d'union entre les deux Canada, migration vers la Nouvelle-Angleterre, etc.). Ainsi, le Nord aurait joué, pour lui, le rôle de la frange pionnière au Canada français, à l'image de la frontière dans le développement de l'Ouest étasunien. Au-delà des éléments constitutifs sur lesquels il s'élabore, le mythe est, selon le géographe lanaudois, avant tout un élément qui mène à l'action.

Évoluant dans le temps, le mythe du Nord, et les conceptions qu'il véhicule, seraient passés subséquemment d'un espace de liberté et d'aventure, au XVIIIe siècle, réalité qui inquiète le clergé et les seigneurs à l'époque des coureurs des bois, à celui de milieu rébarbatif peu propice à l'agriculture, dans la première moitié du XIXe siècle, vision alimentée par la compagnie de la Baie d'Hudson et les entreprises forestières qui cherchaient à repousser le peuplement permanent des territoires au Nord de la vallée du Saint-Laurent (Perron, 2012). Néanmoins, c'est la recomposition tripartite des représentations du Nord entre les années 1870 et 1950 qui interpelle Morissonneau:

\footnotetext{
La terre promise, la mission providentielle, la régénération. Il n’existe pas, entre ces trois composantes, de priorité diachronique, mais une hiérarchie dans l'information. Le lien peut se schématiser de cette façon verbalisée: la providence a assigné aux Canadiens Français la mission de conquérir le Nord qu'elle leur a réservé pour qu'ils y survivent et s'y renforcent (1978: 30).
}

C'est dans cet environnement idéologique que seront organisées les missions de colonisation au Lac-Saint-Jean, dans l'Outaouais, dans les Laurentides et en Abitibi, au début XXe siècle. Selon Morissonneau, si le clergé joue un rôle de premier plan dans la diffusion de ce mythe, les politiciens et les médias y contribuent à maintes occasions. Malgré son analyse des écrits de plusieurs auteurs (François Xavier-Garneau, Antoine Labelle, Arthur Buies, Alphonse Nantel, etc.), Morissonneau aborde assez peu les caractéristiques biophysiques ou humaines du Nord derrière les conceptions du mouvement de colonisation agricole. Contrairement à d'autres géographes, il se concentre principalement sur la construction sociale du mythe du Nord en se référant aux discours des élites religieuses et politiques du temps, en traitant principalement de ce qu'Hamelin appelle le Pré-Nord. Dans son texte de 1982, il précisera sa thèse du mythe du Nord en y adjoignant les images de la nature et de la féminitude. Selon lui, 
autant l'ingénieur que le politicien ou l'écologiste réfère à ces représentations lorsqu'il est question du Nord. Dans cette optique, les Autochtones seraient vus comme des enfants de la nature, intrinsèquement liés au Nord.

Au chapitre V de son livre de 1978, il s'attarde quelque peu sur la localisation ou les frontières du Nord en nommant trois référents principaux utilisés par les auteurs ou acteurs sociaux de l'époque: le Nord continental ou canadien, le Nord québécois (ou péninsulaire) et le Nord de Montréal (partie sud du massif laurentien). Autrement, le Nord mythique des élites y est décrit comme un pays neuf, largement boisé, qui met au défi les individus qui viennent s'y installer à demeure. Les phénomènes du froid et de la présence autochtone sont à peine effleurés dans cette conception du Nord qui a dû laisser pantois les premiers colonisateurs confrontés à la réalité du terrain. Bref, la conception portée par les tenants de la colonisation du Nord compte peu d'information sur le pays «réel» dans la mesure où les attributs géographiques ainsi spécifiés auraient probablement contré l'objectif même du mythe alors proposé, soit rendre le Nord attractif et soutenir des mouvements de populations.

En dépit d'avoir documenté le travail de construction d'un mythe réalisé par les élites afin de soutenir la colonisation agricole du Nord du Québec, l'ouvrage de Morissonneau a essuyé certaines critiques. Il lui a notamment été reproché d'avoir surimposé sans nuance la thèse étasunienne de la frontière sur la situation québécoise, d'avoir sous-estimé le rôle de l'industrialisation dans le peuplement du Nord, ainsi que d'avoir négligé «les situations concrètes» (Séguin, 1979: 75) par son accent excessif sur l'idéologie et le mythe. Nonobstant ces critiques, soulignons que, dans le dernier chapitre de son livre, Morissonneau traite de l'industrie dans le Nord et de son contrôle par les Anglos-Saxons, considérant cette réalité comme une contradiction du mythe du Nord francophone. Par ailleurs, Urbano (1979) soutient, dans un compte rendu de livre, que Morissonneau ne met pas suffisamment l'accent sur la dimension des rapports de classe, le mythe du Nord permettant, avant tout, aux élites du temps de maintenir l'ordre ancien et leur suprématie. En dépit de ces critiques, l'œuvre en question reste une contribution d'envergure à la géographie du Nord, contribution qui est encore aujourd'hui fréquemment citée lorsqu'on jette un regard engagé sur le Plan Nord du gouvernement du Québec. La thèse de Caroline Desbiens se veut, d'une certaine manière, et comme on le verra plus loin, le prolongement des écrits de Christian Morissonneau.

\section{Le Nord et les Autochtones selon Jules Dufour (1941-2017)}

Fortement impliqué dans diverses causes sociales aux échelles québécoise, canadienne et internationale, Jules Dufour connaît également très bien le Nord du Québec. Cet engouement pour le Nord est peut-être lié au fait qu'il a été l'élève d'Hamelin à l’Université Laval, à la fin des années 1960 et au début des années 1970. En plus d'avoir servi comme commissaire à plusieurs reprises pour le Bureau d'audiences publiques en environnement du Québec (BAPE), il a participé aux travaux de la Commission du Nunavik, entre 1999 et 2001, sillonnant chaque village inuit du Québec situé au nord du 55e parallèle (Dufour et Tremblay, 2001). Sa connaissance approfondie de l'Amérique latine a pu également lui permettre de mettre en perspective la question autochtone au Québec. 
Optant pour des postulats très critiques à l'égard de l'économie de marché, la militarisation des sociétés et le sort réservé aux peuples aborigènes, à l'échelle internationale, Dufour réfère souvent aux documents et déclarations de l'Organisation des Nations Unies (ONU) pour en appeler à un monde meilleur, notamment les écrits de l'organisme qui prônent un plus grand respect des nations autochtones, voire leur autodétermination (2007). Dans cette optique, il présente le Nord du Québec comme leur territoire, un territoire périphérique aux yeux des "Blancs», mais doté d'une certaine virginité, c'est-à-dire pas encore spolié de manière généralisée malgré les effets et la menace des grands projets industriels (barrages hydroélectriques, exploitation minière, etc.). La trame de fond de ses travaux et de sa pensée nous paraît correctement résumée dans cet extrait:

\begin{abstract}
Au cours du vingtième siècle, les ressources naturelles des grands espaces situés au Nord du Canada ont été mises à la disposition de l'économie nord-américaine. Une véritable ruée vers les ressources énergétiques, minérales, forestières et fauniques s'est effectuée afin de combler en partie les besoins de l'industrie et du tourisme. Plusieurs fronts pionniers ont permis d'avoir un meilleur accès à ces ressources afin de les extraire et de les transporter vers les centres de production et de consommation situés plus au sud. [...] Cette intégration du Nord à l'économie des grands complexes industriels s'est effectuée le plus souvent au détriment du mode de vie traditionnel des peuples nordiques qui ont dû lutter farouchement pour faire reconnaître leur existence et leurs droits (1996: 234).
\end{abstract}

De manière surprenante, Dufour utilise fréquemment l'expression espace vital, dans ses textes, pour traiter des territoires nordiques habités ou revendiqués par les Autochtones habitant au Québec. Traduction du mot lebensraum cher à Friedrich Ratzel, ce terme de la géographie politique allemande du début du XX $\mathrm{X}^{\mathrm{e}}$ est fortement connoté car, dénaturé par Haushofer à la suite des interprétations du politologue suédois Kjellen, il a servi de justificatif aux visées et actions expansionnistes du IIIe Reich, avant et au cours de la Deuxième Guerre mondiale (Rosière, 2007). Il est difficile d’imaginer que le géographe saguenéen emploie ce terme inconsciemment. Il faut croire qu'il a voulu se faire polémiste afin de mettre l'accent sur l'importance d'une assise territoriale pérenne d'une certaine ampleur pour le maintien des modes de vie autochtones. D’ailleurs, il réalise un calcul assez précis des zones réservées ou redonnées aux Autochtones à l'échelle canadienne, cela, à travers divers traités. Il en conclut que les négociations territoriales peuvent porter fruit dans un contexte démocratique (Dufour, 1993: 270-271).

Désireux de préserver cet «espace vital» pour les nations autochtones, Dufour s'est opposé aux divers projets industriels en milieu nordique, que ce soit le projet Grande-Baleine d'Hydro-Québec (Dufour, 1996) ou, plus récemment, le Plan Nord du gouvernement québécois (Dufour, 2012). De manière originale, son texte sur le défunt projet Grande-Baleine combine son opposition au projet à des recommandations pour un développement durable du Nord et à la survie des "peuples nordiques» (tableau 2). Il a aussi formulé un avis au ministre des Affaires indiennes et du Nord du Canada en faveur de la réhabilitation de la zone minière abandonnée et contaminée entourant Schefferville, à la limite de la Côte-Nord québécoise et du Labrador terre-neuvien (Dufour et Tremblay, 2002). 


\section{Tableau 2 Recommandations de Jules Dufour pour un développement durable du Nord}

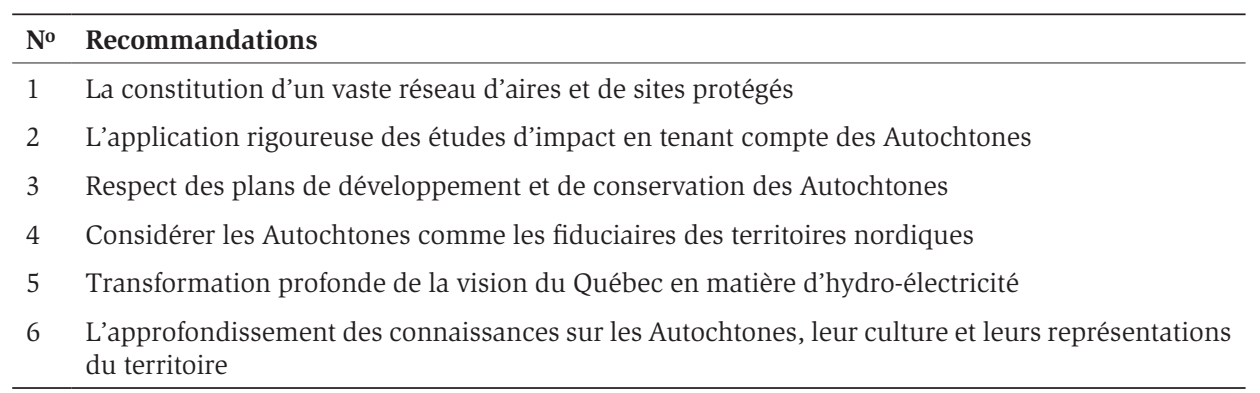

Source: Dufour, 1996: 247-250

L’un des éléments-clés de la vision du Nord de Dufour est probablement son ouverture à l'autodétermination des Inuit "québécois», ouverture exprimée lors du chantier de la Commission du Nunavik (2001), au sein de laquelle il était commissaire. Nationaliste québécois ouvert ou modéré, selon notre compréhension, Dufour y affiche une position généreuse et courageuse, car l'intégrité territoriale du Québec a toujours été défendue avec vigueur par le gouvernement québécois et de nombreux intellectuels (Dorion et Lacasse, 2011). En fait, il s'agit presque d'un tabou respecté même par ceux et celles qui proposent plus d'autonomie aux diverses nations autochtones. Lors du référendum inuit de 2011, les habitants de ce territoire ont, de manière étonnante, rejeté l'option de l'autodétermination. Cette question géopolitique délicate n'a donc pas eu à être débattue de manière approfondie, mais elle resurgira inévitablement dans le futur, le Nunavut et le Kalaallit Nunaat (Groenland), si proches, pouvant servir d'exemples (ou de contre-exemples?).

En rétrospective, dans sa conception pro-autochtone du Nord, Dufour ne s'attarde pas beaucoup aux propriétés du territoire, un peu comme Morissonneau, sauf pour décrire les impacts environnementaux avérés ou projetés des grands projets et réclamer la préservation du milieu, à la faveur des Autochtones et de l'ensemble des Québécois. Néanmoins, il mentionne que le Nunavik est essentiellement un territoire de plaines et de plateaux, passant d'un paysage de taïga à celui de toundra, en remontant vers le nord. Par ailleurs, sa conception du Nord est presque l'antithèse du mythe décrit par Morissonneau. Dufour se représente, en effet, le Nord comme un espace déjà habité, à ne pas perturber, plutôt qu'un territoire vierge à conquérir. Lors de notre recension, nous n'avons pas trouvé de critique précise de ses travaux, possiblement parce ses idées n’ont pas été rassemblées en un livre qui aurait pu faire référence. Toutefois, ses préoccupations pour le Nord et pour les Autochtones, ainsi que son ouverture à leur égard dans les années 1980 et 1990, période de la crise d'Oka et de la controverse sur le dossier Grande-Baleine, apparaissent exceptionnelles par rapport aux positions adoptées ou aux sujets abordés en général par les géographes québécois (Rivard, 2006). 


\title{
Le Nord ou le mythe sous-jacent aux grands projets chez Caroline Desbiens (1969-)
}

La plus jeune des géographes nordistes de notre étude, Caroline Desbiens, se caractérise aussi par une forte sensibilité à la situation et aux revendications des Autochtones. Professeure à l'Université Laval, sa thèse sur les travaux de la BaieJames et, ce qu'elle appelle la «culture de l'hydro-électricité» chez les Québécois, a été publiée en anglais et en français. Détenant une formation multidisciplinaire ayant culminé par un doctorat au département de géographie de l’Université de la ColombieBritannique, les recherches de Desbiens nous apparaissent plus influencées par la vision environnementaliste, très présente sur la Côte Ouest canadienne, que par le nationalisme québécois, ce qui lui offre un certain recul pour décortiquer le mythe de la Baie-James, lequel s'inscrirait plus largement dans le mythe du Nord. Desbiens s'inspire à la fois des courants humaniste et critique, voire postcolonial et féministe, et elle s'appuie sur l'analyse de textes, au plan méthodologique.

Dans son livre Puissance Nord (2015), elle parle d'un mythe du Nord de manière quelque peu semblable à Morissonneau, mais avec davantage de distance critique. Par ailleurs, si elle reconnaît explicitement la parenté de ses travaux avec ceux du géographe lanaudois, elle se réfère fréquemment à l'œuvre d'Hamelin. Dans son analyse préliminaire de ce qu'elle qualifie de «romans de la terre», elle distingue le mythe religieux de la colonisation du Pré-Nord de celui, plus laïque, de la conquête du Nord, une nuance que ne fait pas Christian Morissonneau. Malgré son détour par les textes sur la colonisation agricole, dans ses recherches, elle vise principalement le projet hydroélectrique de la Baie-James et la rhétorique nationaliste et corporatiste qui soutenait, selon elle, la démarche:

\begin{abstract}
Le discours des médias au sujet de la Baie-James reprenait des éléments clefs de la relation qu'entretiennent les Québécois avec le lieu et qui se définit, entre autres, par l'histoire de l'occupation et de l'expansion territoriale via l'agriculture. Si la vie agricole canadienne-française ne pouvait être exportée aussi loin au nord que la Baie-James, l'univers symbolique auquel elle avait donné naissance au fil de quatre siècles de colonisation pouvait être recontextualisé dans un nouvel espace et une nouvelle époque. La répétition constante des idées de terre, de nature et d'identité a servi à intégrer la Baie-James - un territoire historiquement habité et exploité par les Cris-dans le territoire national québécois. La Baie-James offrait un nouvel espace pour la construction de la nation québécoise, mais le discours symbolique employé pour le présenter aux Québécois du Sud était bien connu (2015: 8-9).
\end{abstract}

Prenant, comme Jules Dufour, le parti des nations autochtones, elle dénonce le fait que le territoire du Moyen Nord jamésien ait été considéré comme vide, alors qu’il est l'habitat de plusieurs communautés autochtones, notamment les Cris, les Innus et les Inuit, et ce, depuis des siècles. Desbiens n’hésite pas à qualifier le projet de la Baie-James de néocolonialiste, projet réalisé paradoxalement au moment même où les Québécois cherchent à s'affirmer et à se libérer de leur propre passé de colonisés. Audelà de la prise de contrôle du territoire préconisée par la démarche jamésienne et de sa transformation profonde par Hydro-Québec, par l'intermédiaire de la Société pour le développement de la Baie-James (SEBJ), la géographe reproche l'imposition par les «Blancs» d'une nouvelle vision du Nord qui ne tient pas compte des conceptions plus sensibles des Autochtones. Ces conceptions autochtones seraient, selon toute 
vraisemblance, plus avérées et authentiques, car plus en résonance avec le territoire, alors qu'elles découlent d'une territorialité autre. Selon elle, les Autochtones connaîtraient leur milieu de manière plus fine, observeraient les mouvements des animaux et l'évolution de la végétation au fil des saisons, une vision forgée par le regard du marcheur ou du canoteur. Au contraire, la conception du Nord provenant du Sud est caractérisée par l'utilitarisme, l'utilisation d'images surplombantes provenant de cartes et de photos aériennes ainsi qu'à travers la notion occidentale de perspective, qui rationalise les paysages et leurs composantes (catégorisation, proportions, distances, etc.).

Une autre thèse de Desbiens sur le chantier de la Baie-James concerne sa caractérisation comme étant quasiment raciale ou ethnique, en plus de favoriser la marginalisation des femmes dans des tâches spécifiques, de second niveau. Selon cette géographe, les travailleurs de cet immense projet des années 1970 étaient presque exclusivement des hommes caucasiens et francophones. De surcroît, en dehors des heures de travail, ceux-ci étaient confinés à leurs baraquements, de manière à limiter leurs contacts avec le milieu naturel environnant et les Autochtones. On pourrait aussi ajouter, pour prolonger ses propos, qu'il y a eu une forme de racialisation des gains et des pertes derrière la territorialisation du projet, soit à travers le principe «allant de soi» pour Hydro-Québec, le gouvernement provincial et la majorité des citoyens, de produire de l'électricité pour le Sud en regroupant les retombées négatives au Nord (construction de vastes structures perturbatrices localisées ou sous forme de réseaux linéaires, ennoiement de larges territoires, déplacements à répétition de plusieurs collectivités, problématique du méthylmercure dans les plans d'eau et au sein de la chaîne alimentaire, etc.). Évidemment, la CBJNQ peut être présentée comme un gain relatif pour les Autochtones dans le contexte difficile des bouleversements les affectant, car la CBJNQ reconnaît au moins leur existence et leur accorde certains droits. C'est ce que fait Desbiens en décrivant explicitement cet accord (2015 : 59-64).

D’autre part, Puissance Nord est également assez critique envers le Plan Nord adopté en 2011 par le gouvernement québécois. Selon Desbiens, le programme d'intervention affiche une vision semblable à celle qui a prévalu lors du grand projet de la Baie-James. La géographe emploie d'ailleurs l'expression monstre à deux têtes, dans un texte écrit avec un autre géographe québécois, Étienne Rivard (Rivard et Desbiens, 2011). Le duo fait ici référence aux objectifs apparemment contradictoires du développement minier et du développement durable, objectifs qui sont tous deux énoncés dans la politique. Plus encore, les deux auteurs avancent que, si les Autochtones sont reconnus dans le Plan Nord comme des partenaires pouvant négocier directement avec les compagnies minières ou forestières prévoyant s'implanter à proximité de leurs communautés, diverses inégalités perdurent dans la capacité de le faire entre les nations ayant participé à l'expérience de la CBJNQ et celles qui n'ont pas été en mesure de le faire, par exemple les Innus. À notre avis, ces «Ententes sur les retombées et les avantages» (ERA) manquent aussi de transparence, voire ouvrent la porte à la corruption au sein des conseils de bande.

Plus fondamentalement, Desbiens discute du statut légal des territoires nordiques et de l'avenir des relations entre «Blancs» et Autochtones: 
Si la cogestion territoriale et le partage réel des bénéfices du développement doivent devenir des priorités, la question de la légitimité des revendications du Québec par rapport aux régions nordiques devra être abordée de front et, par conséquent, les imaginations coloniales et les pratiques qu'elles autorisent, être analysées de manière critique. Le contexte d'aujourd'hui offre une occasion de faire les choses différemment et d'injecter une nouvelle signification dans la vision du Nord qu'entretient le Québec du Sud (2015 : 275).

Pour instaurer une véritable collaboration avec les Autochtones et nordiciser le Québec, Desbiens, dans un autre article (2012), propose plusieurs idées à mettre en pratique, idées qui rejoignent largement les recommandations antérieures de Jules Dufour (tableau 3).

\section{Tableau 3 Idées pour le Nord de Caroline Desbiens}

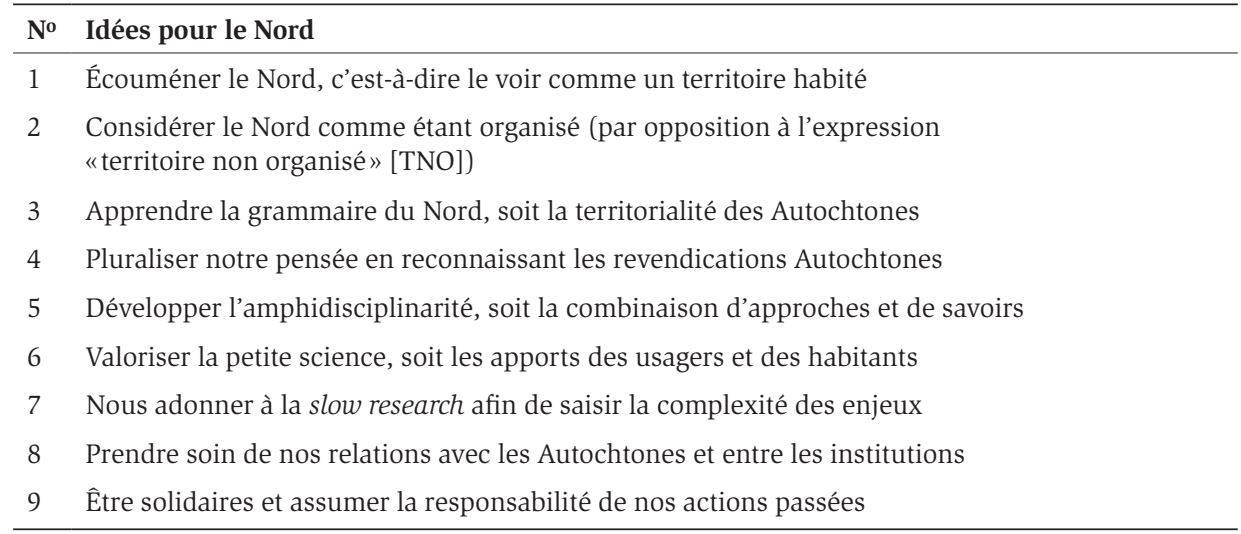

Source: Desbiens, 2012: 645-655

Dans l'ensemble, les écrits de Desbiens ont été bien reçus dans les milieux universitaires. Cependant, deux critiques fondées sur la version originale du document (2013) méritent d'être mentionnées. Un premier commentaire concerne la rareté des références concrètes à la situation des Autochtones, par exemple leur nombre, leur localisation, les impacts qu'ils ont subis, etc. (Armstrong, 2014). Une deuxième critique souligne le manque de description de l'industrie de l'hydroélectricité et du contexte politicoéconomique qui l'englobait à cette époque (Harrison, 2014). Or, malgré ces éventuelles faiblesses, les travaux de Desbiens ont, selon nous, le mérite de tisser des ponts avec les études antérieures des géographes «humains» spécialisés sur le Nord et d'ouvrir de nouvelles perspectives. En atteste sa déconstruction du mythe de la Baie-James posant «l'Hydro-Québécois » en conquérant d'un Moyen Nord sauvage et inhabité de même que ses allusions aux représentations nordiques des Autochtones.

\section{La comparaison des visions et idées des auteurs}

Notre présentation des conceptions du Nord québécois des auteurs ciblés étant terminée, tentons maintenant de les comparer (tableau 4). En premier lieu, il semble que les quatre spécialistes sondés aient mis en parallèle le Nord et la question autochtone, soit deux éléments qui leur paraissent inséparables conceptuellement. 
Par exemple, les auteurs se montrent sensibles aux réalités sociales et économiques difficiles et aux revendications des Autochtones, quoique Morissonneau ne soit pas très prolifique sur ce dernier sujet. Cette vision d'un Nord habité qu'il convient de mieux connaître et de respecter, qui est adoptée par les géographes mis en exergue, paraît en rupture avec la conception de type terra nullius (Rivard, 2006) ou «d'espace instrumentalisé» (Lasserre, 2003) qui prévaudrait chez de nombreux décideurs et au sein de la population. Incidemment, cela nous invite à repenser en profondeur le concept géographique d'écoumène, c'est-à-dire réfléchir aux conditions en vertu desquelles un territoire peut être considéré comme habité.

\section{Tableau 4 Comparaison des conceptions du Nord chez les auteurs}

\begin{tabular}{|c|c|c|}
\hline Auteurs & Caractéristiques & Paradigme associé \\
\hline $\begin{array}{l}\text { Louis-Edmond } \\
\text { Hamelin }\end{array}$ & $\begin{array}{l}\text { - Territoire froid } \\
\text { - Accès difficile } \\
\text { - Peuplement faible et dispersé } \\
\text { - Conditions de vie misérables } \\
\text { - Territoire autochtone à connaître et à respecter }\end{array}$ & $\begin{array}{l}\text { - Positivisme évoluant } \\
\text { vers l'humanisme }\end{array}$ \\
\hline $\begin{array}{l}\text { Christian } \\
\text { Morissonneau }\end{array}$ & $\begin{array}{l}\text { - Territoire soumis au phénomène de frontière } \\
\text { - Territoire des futures régions périphériques } \\
\text { - Autochtones comme enfants de la nature }\end{array}$ & $\begin{array}{l}\text { - Humanisme se } \\
\text { combinant à des } \\
\text { élans postcoloniaux }\end{array}$ \\
\hline $\begin{array}{l}\text { Jules } \\
\text { Dufour }\end{array}$ & $\begin{array}{l}\text { - Territoire habité } \\
\text { - Territoire spolié } \\
\text { - Terres revendiquées } \\
\text { - Autochtones à respecter comme fiduciaires }{ }^{4} \text { des lieux }\end{array}$ & $\begin{array}{l}\text { - Humanisme se } \\
\text { combinant à } \\
\text { l'approche critique }\end{array}$ \\
\hline $\begin{array}{l}\text { Caroline } \\
\text { Desbiens }\end{array}$ & $\begin{array}{l}\text { - Territoire habité } \\
\text { - Territoire spolié } \\
\text { - Terres revendiquées } \\
\text { - Autochtones à respecter comme propriétaires des lieux }\end{array}$ & $\begin{array}{l}\text { - Humanisme se } \\
\text { combinant à } \\
\text { l'approche critique }\end{array}$ \\
\hline
\end{tabular}

Conception: Simard, 2017

Au demeurant, Hamelin, Dufour et Desbiens soulignent l'immense différence entre les représentations territoriales des «sudistes» et celles des Autochtones, dans les limites de ce que nous savons de ces dernières. Leur intérêt envers les conceptions des Autochtones s'affiche comme positif en soi, mais il confirme aussi l'existence d'un clivage culturel important. Des points de vue de ces trois auteurs, et du nôtre, il est essentiel d'approfondir ce chantier actuellement peu documenté car la compréhension, ou le partage éventuel, des schémas mentaux de référence constituent autant de conditions du respect de l'autre, d'un vivre-ensemble harmonieux et de la négociation d'ententes consensuelles répondant aux besoins de toutes les parties prenantes.

En deuxième lieu, si l'on tente d'établir des différences entre les conceptions portées ou décrites par les quatre géographes étudiés, on constate qu'Hamelin et Dufour se tiennent plus près du terrain. Ils traitent plus fréquemment du «pays réel» et de ses propriétés physiques, peut-être parce qu’ils sont ceux, parmi les géographes de notre échantillon, qui ont le plus fréquenté le Nord au cours de leur carrière respective.

4 Le terme fiduciaire signifie ici «le légataire chargé de restituer les biens en vertu d'un fidéicommis» (TPSGC, 2017). 
Incidemment, Hamelin se rapproche, au départ, des visions concrètes des géographes physiques interpellés par le Nord, dont nous avons peu parlé dans ce texte (Vallée, 2011). Toutefois, Hamelin évoluera rapidement, rappelons-le, vers une conception plus humaniste du Nord, conception qui deviendra en fait sa marque de commerce. Il en va de même pour Jules Dufour. Pour leur part, Christian Morissonneau et Caroline Desbiens travaillent à un plus haut niveau d'abstraction, un niveau qu'Hamelin atteindra également par moments, notamment à travers sa notion de nordicité mentale et ses travaux en linguistique. D'un point de vue épistémologique, nos quatre auteurs cadrent tous, à bien des égards, à l'intérieur de l'approche humaniste, possiblement en partie à cause de l'influence des travaux de ce même Hamelin, à partir des années 1980. Néanmoins, Dufour et Desbiens intègrent aussi à leurs analyses des postulats de la géographie critique, et même Morissonneau qui fait en partie de la critique postcoloniale avant la lettre.

Pour revenir sur l'opposition attributs concrets versus l'imaginaire, Morissonneau et Desbiens abordent à peine des caractéristiques physicospatiales du territoire nordique. Cela tient peut-être à leur méthode qui les amène à faire des analyses de discours, donc à étudier principalement les visions des autres, ou à leur intérêt premier sinon à leur objet spécifique. Conséquemment, ils s'attardent en majeure partie au processus de construction des conceptions territoriales par les acteurs sociaux, ainsi qu'à l'intentionnalité que celles-ci contiennent. Par le truchement de leurs textes, on découvre néanmoins leurs propres conceptions du Québec nordique, soit une conception plutôt nationaliste ou régionaliste, chez Morissonneau, soit une vision plutôt autochtoniste et environnementaliste, chez Desbiens. Cette différence tient peut-être à une question de génération, les enjeux de société des années 1970

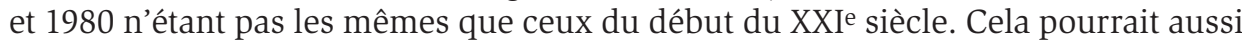
être associé aux regards marqués par le sexe des auteurs en question, dans la mesure où les écrits de Caroline Desbiens se distinguent des auteurs masculins, tout en étant représentatifs d'une vision féminine ou féministe plus largement partagée.

\section{Conclusion}

Au cours des six dernières décennies, relativement peu de "géographes humains » québécois se sont investis durablement sur le Nord, la nordicité, voire l'hiver, ce qui est troublant considérant qu'il s'agit de caractéristiques fondamentales de notre territoire. Fernand Harvey (1994) faisait le même constat du faible intérêt pour le Nord chez les historiens, dans les années 1990. Malgré tout, on observe une continuité dans le temps des recherches géographiques sur les aspects humains du Nord et une filiation certaine des approches et des idées chez les quelques spécialistes engagés sur ce terrain, sous l'influence notable de Louis-Edmond Hamelin. D’ailleurs, cette influence est également sensible dans d'autres champs disciplinaires, voire dans une partie du grand public. De plus, on observe que l'engouement pour les régions périphériques, les Autochtones et le Nord québécois est bien vivant chez plusieurs jeunes géographes québécois, par exemple Laurie Guimond, Étienne Rivard ou Pierre-Louis Têtu. Peuton dire pour autant que tous ces «nordistes» forment une école de pensée? À tout le moins, il est permis d'affirmer qu'il s’agit d'une filière importante de la géographie humaine québécoise, filière caractérisée principalement par l'approche humaniste. 
En effet, les écrits des géographes considérés ici s’inscrivent clairement dans un courant humaniste, additionné de divers éléments critiques, ce qui les rapproche des travaux des anthropologues (Geneviève Brisson), des sociologues (Gérard Duhaime) ou des historiens (Camil Girard). Cette position rejoint la tendance plus générale à l'interpénétration des paradigmes culturaliste et radical, au sein de la géographie humaine. Dans les faits, ces diverses recherches contribuent à faire du Nord québécois, ou canadien, un chantier pluridisciplinaire de plus en plus fécond. Il paraît donc approprié de mettre au second plan les comptabilités ou les analyses sur les contributions respectives des chercheurs en fonction de disciplines scientifiques spécifiques. Plus largement, la compréhension des conceptions du monde des différents auteurs et acteurs est une ouverture importante vers des possibilités de dialogues plus constructifs et d'actions plus informées et respectueuses envers les populations et l'environnement, en milieu nordique. Même le Plan Nord du gouvernement du Québec n'a pu faire l'économie de chapitres sur les réalités et les droits des Autochtones malgré son parti-pris évident pour le développement industriel.

Somme toute, sur les plans idéologique et politique, la «question nationale québécoise» n’est pas véritablement soulevée par les auteurs, du moins dans sa forme contemporaine. En effet, le mythe du Nord décrit par Morissonneau illustre l'élite d'une autre époque préoccupée par la survivance de la «race canadienne-française». Indépendamment de cela, les géographes de l'échantillon ne regardent pas le Nord du Québec en fonction de lunettes nationalistes, selon nos impressions. De surcroît, Desbiens semble même déplorer ce nationalisme dans la mesure où il se positionne en arrière-plan du mythe de l'Hydro-Québécois qui est à la base des travaux de la BaieJames, travaux qu'elle juge fort dommageables pour les nations autochtones. Est-ce que ce faible nationalisme québécois dans les textes étudiés et les visions globalement autochtonistes du Nord répertoriées sont symptomatiques de notre époque où le nationalisme semble en baisse de popularité au Québec, ou s'agit-il d'un hasard dû à la sélection des auteurs? Dans l'éventualité de sa véracité, ce constat du nationalisme à la baisse nous paraît significatif, car il laisse poindre une ère où les revendications territoriales autochtones ne seront plus évaluées simplement à l'aune de l'intégrité territoriale du Québec. Néanmoins, la question politique demeure digne d'intérêt, ne serait-ce que pour l'État québécois. À cet égard, est-ce que 60 ans de présence dans le Nord du gouvernement du Québec et d'intervenants francophones en santé et en éducation auront suffi à donner le goût aux Eeyouch ou aux Nunavimmiut de maintenir un certain arrimage à la province, en cas d'obtention d'un statut particulier? 


\section{Bibliographie}

ALLARD, Michel et LEMAY, Mickaël (dir.) (2013) Le Nunavik et le Nunatsiavut: de la science aux politiques publiques. Une étude intégrée d'impact régional des changements climatiques et de la modernisation. Québec, ArcticNet.

ARMSTRONG, Christopher (2014) Book review: Power from the North. Territory, identity and the culture of hydroelectricity in Quebec. Environmental History, vol. 19, no4, p. 771-773.

BAILLY, Antoine, DORELL, Gérard, RACINE, Jean-Bernard et VILLENEUVE, Paul (dir.) (1992) États-Unis, Canada. Paris, Belin et Montpellier, Reclus.

BONE, Robert M. (2016) The Canadian North. Issues and challenges. Toronto, Oxford University Press.

CANOBBIO, Éric (2009) Géopolitique d'une ambition inuite. Le Québec face à son destin nordique. Québec, Septentrion.

DESBIENS, Caroline (2012) 10 idées pour le Nord: un manifeste pour la nordicité. Cahiers de géographie du Québec, vol.56, no159, p. 643-659.

DESBIENS, Caroline (2014) Power from the North. Territory, identity, and the culture of hydroelectricity in Quebec. Vancouver, University of British Columbia Press.

DESBIENS, Caroline (2015) Puissance Nord. Territoire, identité et culture de l'hydroélectricité au Québec. Québec, Presses de l'Université Laval.

DORION, Henri et LACASSE, Jean-Paul (2011) Le Québec: territoire incertain. Québec, Septentrion.

DROIT, Roger-Pol (2014) Au Nord, le mythe de la sagesse glacée. Le Devoir, Montréal, 27 juillet 2014 [En ligne]. www. lemonde.fr/livres/article/2014/07/28/ au-nord-le-mythe-de-la-sagesseglacee_4458884_3260.html

DUFOUR, Jules (1993) Les revendications territoriales des peuples autochtones au Québec. Cahiers de géographie du Québec, vol. 37, nº101, p. 263-290.
DUFOUR, Jules (1996) Le projet GrandeBaleine et l'avenir des peuples autochtones au Québec. Cahiers de géographie du Québec, vol.40, n¹10, p. 233-252.

DUFOUR, Jules (2012) Plan Nord: la dérogation aux droits ancestraux et fonciers des autochtones. Mondialisation.ca [En ligne]. http://www.mondialisation. ca/plan-Nord-la-d-rogation-auxdroits-ancestraux-et-fonciers-desautochtones $/ 30730$ ?print $=1$

DUFOUR, Jules et CÔTÉ, Claude P. (1983) Profil régional du Nord du Québec. Québec, Office de planification et de développement économique du Québec.

DUFOUR, Jules et TREMBLAY, Marc-Adélard (2001) La Commission du Nunavik: les leçons d'une expérience unique et extraordinaire. Québec, Commission du Nunavik.

DUFOUR, Jules et TREMBLAY, Marc-Adélard (2002) La zone minière de Schefferville et sa réhabilitation. Avis formulé à l'intention du ministre des Affaires indiennes et du Nord du Canada. Québec, Les classiques des sciences sociales.

DUHAIME, Gérard (2010) Images du Nord et de quelques héros. Recherches sociographiques, vol.51, n³, p. 445-459.

DUHAIME, Gérard et GODMAIRE, Anne (2002) Les modèles de développement du nord. Analyse exploratoire au Québec isolé. Recherches sociographiques, vol. 43, no2, p. 329-351.

DUHAIME, Gérard et ROBICHAUD, Véronique (2010) L'économie du Nunavik entre 1983 et 2003. Recherches sociographiques, vol. 51, nos $1-2$, p. 45-73.

HAMELIN, Louis-Edmond (1975) Nordicité canadienne. Montréal, Hurtubise HMH.

HAMELIN, Louis-Edmond (2000) Le Nord et l'hiver dans l'hémisphère boréal. Cahiers de géographie du Québec, vol.44, nº121, p. 5-25. 
HAMELIN, Louis-Edmond (2006) L'âme de la terre. Parcours d'un géographe. Montréal, MultiMondes.

HARRISON, Conor M. (2014) Book review: Power from the North. Territory, identity and the culture of hydroelectricity in Quebec. Cultural Geographies, vol.21, $\mathrm{n}^{\circ} 4$, p. 745-753.

HARVEY, Fernand (1994) L'historiographie du Nord du Québec. Recherches sociographiques, vol.35, n³, p. 373-420.

LASSERRE, Frédéric (2003) L'eau, la forêt, les barrages du Nord du Québec. Un territoire instrumentalisé. Dans Frédéric Lasserre et Aline Lechaume (dir.) Le territoire pensé. Géographie des représentations territoriales. Québec, Presses de l'Université du Québec, p. 13-28.

LASSERRE, Frédéric (2009) Les aménagements hydroélectriques du Québec: le renouveau des grands projets. Géocarrefour, vol. 84, nos 1-2, p. 11-18.

MARTIN, Thibault (2003) De la banquise au congélateur. Mondialisation et culture au Nunavik. Québec, Presses de l'Université Laval.

MARTIN, Thibault (2010) Vers la fin du "contrat colonial moderne»? Le cas des ententes hydroélectriques au Québec et au Manitoba. Globe, vol. 13, n² 2, p. 125-150.

MORISSET, Jean (1985) L'identité usurpée 1) l'Amérique écartée. Montréal, Nouvelle Optique.

MORISSONNEAU, Christian (1978) La terre promise: le mythe du Nord québécois. Montréal, Hurtubise HMH.

MORISSONNEAU, Christian (1982) Le Nord qui est nature qui est féminitude. Cahiers de géographie du Québec, vol.26, nº68, p. 241-246.

MORTON, William L. (1970) The North in Canadian historiography. Transactions of the Royal Society of Canada, série IV, no8, p. 31-40.

ONU (ORGANISATION DES NATIONS UNIES) (2007) Déclaration des Nations Unies sur les droits des nations autochtones. New York, 107 e séance plénière, 13 septembre 2007 [En ligne]. http://www.un.org/esa/ socdev/unpfii/documents/DRIPS_fr.pdf
PELLETIER, Gérard (1986) Compte rendu: L'identité usurpée 1) l'Amérique écartée. Cahiers de géographie du Québec, vol.30, no79, p. 83-84.

PERRON, Normand (2012) Le Nord-duQuébec: un environnement à découvrir. Dans Girard, Réjean (dir.) Histoire du Nord-du-Québec. Québec, Presses de l'Université Laval, p. 17-38.

RIVARD, Étienne (2006) Terra Nullius ou géographie de l'absence: les géographes québécois et la question autochtone au pays. Cahiers de géographie du Québec, vol. 50, no141, p. 385-392.

RIVARD, Étienne et DESBIENS, Caroline (2011) Le Plan Nord, monstre à deux têtes et autres chimères géographiques. Recherches amérindiennes au Québec, vol. 41, nº1, p. 83-89.

ROSIÈRE, Stéphane (2007) Comprendre l'espace politique. L'Espace politique, vol. no 1 [En ligne]. http://journals. openedition.org/espacepolitique/223

SÉGUIN, Normand (1979) Compte rendu: La terre promise, le mythe du Nord québécois. Revue d'histoire de l'Amérique française, vol. 33, n³, p. 473-476.

TPSGC (TRAVAUX PUBLICS ET SERVICES GOUVERNEMENTAUX CANADA) (2017)

Fiduciaire. Terminum Plus. La banque de données terminologiques et linguistiques $d u$ gouvernement du Canada [En ligne]. www.btb.termiumplus.gc.ca/tpv2alpha/ alpha-fra.html?lang=fra\&i $=1 \&$ srchtxt $=$ fid uciaire\&index $=$ alt\&codom 2 nd_wet $=1$

URBANO, Henrique (1979) Compte rendu: La terre promise, le mythe du Nord québécois. Recherches sociographiques, vol. 20, nº1, p. 130-131.

VALLÉE, Pierre (2011) À la conquête du Grand Nord. Le Centre d'études nordiques entend contribuer à l'ambitieux Plan Nord. Le Devoir, Cahier spécial, 6 et 7 août 2011 [En ligne]. http://www.cen.ulaval. $\mathrm{ca} /$ document/cahiercendevoir.pdf 